\title{
The Value of Whole-tumor CT Texture Analysis as a Quantitative Biomarker in the Identification of Solid Renal Tumors
}

\section{Xiaoyan Meng}

Tongji Hospital, Tongji Medical College, Huazhong University of Science and Technology

Shichao Li

Tongji Hospital, Tongji Medical College, Huazhong University of Science and Technology

\section{Cui Feng}

Tongji Hospital, Tongji Medical College, Huazhong University of Science and Technology

Daoyu Hu

Tongji Hospital, Tongji Medical College, Huazhong University of Science and Technology

Zhen li

Tongji Hospital, Tongji Medical College, Huazhong University of Science and Technology

Yonghua Niu ( $\square$ nyhheiliger@163.com )

Tongji Hospital, Tongji Medical College, Huazhong University of Science and Technology

\section{Research Article}

Keywords: renal tumor, computed tomography, X-ray, texture analysis

Posted Date: February 1st, 2022

DOI: https://doi.org/10.21203/rs.3.rs-1276439/v1

License: () (1) This work is licensed under a Creative Commons Attribution 4.0 International License. Read Full License 


\section{Abstract}

Background: Renal tumors are very common in the urinary system. Preoperative diagnosis for the renal tumor is very important for postoperative prognosis evaluation, surgical method selection, and follow-up observation. This study was to evaluate the value of the whole-tumor CT texture analysis for identification of solid renal tumors without hemorrhage, necrotic or cystic changes.

Methods: 128 patients were included and successfully performed contrast-enhanced CT examinations. All cases were pathologically confirmed. The whole-tumor texture analysis was performed by manually drawn the whole tumor layer by layer, and texture parameters were recorded. The independent-sample $t$ test and Mann-Whitney $U$ test were used to compare the differences, and the receiver operating characteristic (ROC) curve analysis was used to determine the diagnostic efficiency.

Results: With the ROC analysis, 75th, inhomogeneity, and kurtosis provided better diagnostic performance for discriminating cCRCC from chRCC in the arterial phase than those in venous phase ( $p \bigotimes 0.05)$. The entropy performed excellent performance in discriminating ccRCC from chRCC. Kurtosis and entropy performed high sensitivity (91\%) and moderate specificity (74\%) in discriminating RO from chRCC in the arterial phase. The inhomogeneity of ccRCC was significantly higher than that of RO ( $p \otimes 0.05)$, while other parameters showed limited help.

Conclusions: The whole-tumor CT texture analysis could be a useful tool for discriminating solid renal tumor, while provided limited help in distinguishing RO from ccRCC which without hemorrhage, necrotic or cystic changes.

\section{Background}

Renal tumors are very common in the urinary system. Approximately $90 \%$ of renal tumors are renal cell carcinoma (RCC)[1], which originates from renal cortical cell and the three main types are clear cell RCC (ccRCC,75\%), papillary RCC (pRCC,15\%), and chromophobe RCC (chRCC,5\%). According to GLOBCAN 2018, 403,262 new kidney tumor patients were diagnosed all over the world[2]. The remaining solid renal tumors include renal oncocytoma (OR), angiomyolipoma, adenoma, etc. Preoperative diagnosis for the renal tumor is very important for postoperative prognosis evaluation, surgical method selection, and follow-up observation.

CT texture analysis (CTTA) provides an assessment of the volumetric tumor heterogeneity by analyzing the distribution and association with pixel or voxel gray level in the image, detecting the difference in the tissue that is invisible by naked eye. It has been widely used in tumor diagnosis, overall survival, response evaluation, predicting relevant histologic, and protein biomarkers[3-5]. In terms of the renal tumor, CTTA has emerged as a promising method for differentiating cCRCC from pRCC also for prediction of Fuhrman grading $[4,6,7]$ and histogram-based features performed as a promising tool for differentiating RCC subtype from RO[8].

However, none of the above studies mentioned the exclusion of ccRCC with necrosis and cystic. Histologic tumor necrosis is an independent prognostic indicator for ccRCC and papillary RCC[7]. As we all know, about more than $50 \%$ of ccRCC associates with hemorrhage and necrosis, but it still has the challenge to diagnose accurately ccRCC without these changes. Also, chRCC tends to show lower enhancement than cCRCC and more homogenous, but there are substantial overlaps in enhancement characteristics with ccRCC[9]. RO is the second common benign renal tumor, and it is difficult to distinguish it from renal cell carcinoma on imaging. What's more, RO and chRCC have strong overlaps in the imaging findings because of sharing the cell of origin[4]. In addition, chRCC exhibits a 10\% mortality while RO is benign, also RO with nuclear atypia is easily misdiagnosed as chRCC[10], so preoperative diagnosis is crucial.

The purpose of this study was to evaluate the value of the whole-tumor CT texture analysis for the identification of solid renal tumor without hemorrhage, necrotic or cystic changes, including CCRCC, chRCC, and RO. 


\section{Materials And Methods}

\section{Patients}

This retrospective study was approved by our IRB and written informed consent was waived. Retrospectively review of our hospital reporting system between July 2012 and August 2017 to identify patients according to the inclusion criteria:1) all patients were confirmed by surgical histology (pathologically confirmed as CcRCC, chRCC, and RO) and had no other therapeutic measures before the examination;2) the time interval between the contrast-enhanced CT examination and surgery was less than one month;3) there was no obvious artifacts or breathing motion artifacts on the images; 4) tumors without obvious hemorrhage, necrotic or cystic changes. Finally, 128 patients ( 77 ccRCCs, 32chRCCs, and 19 ROs) were enrolled in this study (Fig. 1).

\section{CT examination}

All patients successfully performed contrast-enhanced CT examinations using the HD 750 MDCT scanner (High Discovery 750, HD750, GE Medical System, USA) and a 64-slice CT scanner (Lightspeed VCT, GE Medical System, USA). The scan included three phases of the arterial phase, venous phase, and delayed phase. All patients were performed the examination with a supine position and feet first, scanning covered the entire renal region and each phase of the scan was completed during a single breath-hold. Nonionic contrast media (iopromide, Ultravist 370; Schering, Berlin, Germany) through the 18gauge (18-G) angiographic catheter inserted into an antecubital vein before the examination, and the rate of injection was 3$4 \mathrm{~mL} / \mathrm{s}$ by using automatic dual-flow high pressure injector (Stellant; Medrad, Indianola, PA). The total contrast media dosage varied according to the patient weight $(1.5 \mathrm{~mL} / \mathrm{kg})$ and $20 \mathrm{~mL}$ saline solution followed.

Images were acquired with a helical acquisition and with the parameters: $120 \mathrm{kv}$, automatic MA, helical pitch of 0.983 , rotation speed of $0.6 \mathrm{~s}$, slice thickness and interval of $5 \mathrm{~mm} / 5 \mathrm{~mm}$ and the reconstruction slice thickness of $1.25 \mathrm{~mm}$. The arterial phase was performed by automatically triggering scan mode by monitoring the aortic density value (120HU), the venous phase was performed at a delay of 25 seconds after the arterial phase scanning and the delay phase was performed 3 minutes later.

\section{Imaging analysis}

All DICOM data transformed from the PACS to personal computer and texture analysis performed with a developed software (Fire Voxel, New York University, New York, USA). A radiologist (SC. L. ,7 years of work experience in abdominal imaging diagnosis) manually drawn the region of interests (ROIs) for the whole tumor on all layers along the edge of the lesion as large as possible avoiding perirenal fat. Then each layer of the ROI fused to get the whole tumor information and automatically generated the texture analysis parameters, including mean, inhomogeneity, skewness, kurtosis and entropy, and then calculated 25th, 50th, 75th with statistical software.

\section{Statistical analysis}

Statistical analyses were completely performed using SPPS 22.0 (Chicago, IL) and MedCalc (MedCalc Software, Mariakerke, Belgium) statistical software for Windows. All the texture analysis parameters were recorded as mean \pm standard deviation. The independent-sample $t$ test and Mann-Whitney $U$ test were performed to compare the parameters mean, 25th, 50th, 75th, inhomogeneity, skewness, kurtosis and entropy between ccRCC and chRCC, ccRCC and RO, chRCC and RO both in arterial phase and venous phase. Receiver operating characteristic curve (ROC) analysis was performed to calculate the diagnostic accuracy of the parameters and recorded cutoff value, sensitivity and specificity, also compared the area under the receiver operating characteristic curve (AUC) of parameter in arterial phase with in venous phase. $P$ value below 0.05 indicated a statistically significant difference.

\section{Results}


Of the 128 patients including 77 ccRCCs (M: $F=54: 23$, mean age 47.5 years), 32chRCCs (M: $F=17: 15$, mean age 48.4 years) and 19 ROs (M: $F=12: 7$, mean age 53.5 years), the value of mean, 25th, 50th, 75th, inhomogeneity, skewness, kurtosis, and entropy were shown in Table 1, the $p$ values and AUCs were showed in Table 2, Table 3, and Fig. 2 respectively.

Table 1

Texture analysis parameters of the three groups of solid renal tumors in arterial and venous phases

\begin{tabular}{|llllllllll|}
\hline & & mean & 25th & 50th & 75th & Inhomogeneity & Skewness & Kurtosis & Entropy \\
\hline $\begin{array}{l}\text { Arterial } \\
\text { phase }\end{array}$ & ccRCC & $127 \pm 50$ & $91 \pm 43$ & $125 \pm 50$ & $159 \pm 54$ & $0.044 \pm 0.011$ & $-0.20 \pm 0.48$ & $0.70 \pm 1.35$ & $3.77 \pm 0.21$ \\
\cline { 2 - 10 } & chRCC & $83 \pm 24$ & $61 \pm 22$ & $80 \pm 24$ & $98 \pm 26$ & $0.028 \pm 0.007$ & $-0.71 \pm 1.12$ & $4.97 \pm 6.91$ & $3.25 \pm 0.29$ \\
\cline { 2 - 10 } & RO & $114 \pm 61$ & $84 \pm 52$ & $111 \pm 61$ & $135 \pm 67$ & $0.034 \pm 0.010$ & $-0.30 \pm 0.48$ & $1.24 \pm 1.50$ & $3.65 \pm 0.29$ \\
\hline $\begin{array}{l}\text { Venous } \\
\text { phase }\end{array}$ & ccRCC & $140 \pm 52$ & $106 \pm 44$ & $138 \pm 51$ & $164 \pm 56$ & $0.038 \pm 0.011$ & $-0.47 \pm 0.46$ & $1.40 \pm 1.89$ & $3.72 \pm 0.26$ \\
& chRCC & $100 \pm 46$ & $77 \pm 44$ & $97 \pm 47$ & $115 \pm 48$ & $0.028 \pm 0.007$ & $-0.33 \pm 0.70$ & $3.65 \pm 2.83$ & $3.22 \pm 0.35$ \\
\cline { 2 - 10 } & RO & $130 \pm 49$ & $103 \pm 46$ & $128 \pm 49$ & $148 \pm 51$ & $0.031 \pm 0.010$ & $-0.70 \pm 0.56$ & $2.37 \pm 2.53$ & $3.64 \pm 0.23$ \\
\hline
\end{tabular}

Table 2

The $p$ values of texture analysis parameters in differentiating the three subtypes of renal solid tumors in arterial and venous phase.

\begin{tabular}{|c|c|c|c|c|c|c|}
\hline & \multicolumn{3}{|c|}{ Arterial phase } & \multicolumn{3}{|c|}{ Venous phase } \\
\hline & $\begin{array}{l}\text { ccRCC vs } \\
\text { chRCC }\end{array}$ & $\begin{array}{l}\text { ccRCC vs } \\
\text { RO }\end{array}$ & $\begin{array}{l}\text { RO vs } \\
\text { chRCC }\end{array}$ & $\begin{array}{l}\text { ccRCC vs } \\
\text { chRCC }\end{array}$ & $\begin{array}{l}\text { ccRCC vs } \\
\text { RO }\end{array}$ & $\begin{array}{l}\text { RO vs } \\
\text { chRCC }\end{array}$ \\
\hline mean & $\otimes 0.01$ & 0.24 & 0.13 & $\triangle 0.01$ & 0.85 & 0.07 \\
\hline 25th & $\varangle 0.01$ & 0.98 & 0.21 & $\triangle 0.01$ & 1.00 & 0.10 \\
\hline 50th & $\nabla 0.01$ & 0.88 & 0.11 & $\triangle 0.01$ & 0.90 & 0.06 \\
\hline 75th & $₫ 0.01$ & 0.55 & 0.07 & $₫ 0.01$ & 0.70 & 0.06 \\
\hline Inhomogeneity & $\varangle 0.01$ & $₫ 0.01$ & 0.12 & $\llbracket 0.01$ & $\varangle 0.01$ & 0.62 \\
\hline Skewness & 0.88 & 0.81 & 0.65 & 0.21 & 0.11 & 0.02 \\
\hline Kurtosis & $\varangle 0.01$ & 0.40 & 0.01 & $\varangle 0.01$ & 0.34 & 0.26 \\
\hline Entropy & $\otimes 0.01$ & 0.05 & $\bowtie 0.01$ & $\varangle 0.01$ & 0.55 & $\triangle 0.01$ \\
\hline
\end{tabular}


Table 3

Discrimination power (AUC) of texture analysis parameters in differentiating the three subtypes of renal solid tumors in arterial and venous phase.

\begin{tabular}{|c|c|c|c|c|c|c|}
\hline & & arterial phase & & venous phase & & \\
\hline & & $\begin{array}{l}\text { AUC (sensitivity, } \\
\text { specificity) }\end{array}$ & $\begin{array}{l}\text { cut-off } \\
\text { value }\end{array}$ & $\begin{array}{l}\text { AUC (sensitivity, } \\
\text { specificity) }\end{array}$ & $\begin{array}{l}\text { cut-off } \\
\text { value }\end{array}$ & $p$ \\
\hline \multirow{7}{*}{$\begin{array}{l}\text { ccRCC vs } \\
\text { chRCC }\end{array}$} & mean & $0.82(71 \%, 83 \%)$ & 100 & $0.78(81 \%, 67 \%)$ & 94 & 0.13 \\
\hline & 25th & $0.76(65 \%, 78 \%)$ & 70 & $0.74(78 \%, 64 \%)$ & 71 & 0.60 \\
\hline & 50th & $0.83(78 \%, 78 \%)$ & 93 & $0.78(82 \%, 67 \%)$ & 91 & 0.09 \\
\hline & 75th & $0.88(79 \%, 86 \%)^{*}$ & 119 & $0.79(81 \%, 70 \%)^{*}$ & 114 & $\nabla 0.01$ \\
\hline & Inhomogeneity & $0.88(82 \%, 81 \%)^{*}$ & 0.033 & $0.79(75 \%, 72 \%)^{*}$ & 0.031 & $\varangle 0.01$ \\
\hline & Kurtosis & $0.93(87 \%, 92 \%)^{*}$ & 1.31 & $0.81(73 \%, 78 \%)^{*}$ & 1.87 & $\varangle 0.01$ \\
\hline & Entropy & $0.95(91 \%, 89 \%)$ & 3.54 & $0.91(83 \%, 86 \%)$ & 3.56 & 0.05 \\
\hline ccRCC vs RO & Inhomogeneity & $0.77(61 \%, 90 \%)$ & 0.039 & $0.66(92 \%, 47 \%)$ & 0.025 & 0.18 \\
\hline \multirow[t]{2}{*}{ RO vs chRCC } & Kurtosis & $0.84(92 \%, 74 \%)$ & 1.34 & $0.66(75 \%, 58 \%)$ & 1.92 & 0.09 \\
\hline & Entropy & $0.85(92 \%, 74 \%)$ & 3.56 & $0.84(97 \%, 58 \%)$ & 3.62 & 0.92 \\
\hline
\end{tabular}

\section{Ccrce Vs Chrce}

Mean, 25th, 50th, 75th, inhomogeneity, and entropy of ccRCC were significantly higher than that of chRCC, while the kurtosis of ccRCC was significantly lower than that of $\operatorname{chRCC}(p<0.05$ for all). The AUCs of 75th, inhomogeneity, and kurtosis in the arterial phase $(0.88,0.90$ and 0.92$)$ were significantly higher than those in the venous phase $(0.79,0.81$ and 0.80$)$, respectively (all $p \llbracket 0.05$ ). The AUCs of mean, 25th, and 50th in the arterial phase was higher than those in the venous, but the differences were not statistically significant ( $p \bigotimes 0.05)$. The entropy displayed the highest diagnostic performance with AUC, sensitivity, and specificity of $0.95,91 \%$, and $89 \%$ in the arterial phase, and $0.91,83 \%$, and $86 \%$ in the venous phase, respectively.

\section{Ccrcc Vs Ro}

The inhomogeneity of RO was significantly lower than that of cCRCC both in the arterial phase and venous phase, with the AUC of 0.77 (sensitivity, 61\%; specificity, 90\%) and 0.66 (sensitivity, 92\%; specificity, 47\%). The AUC in the arterial phase was higher than that in the venous phase, however, the difference was not significantly significant $(p=0.18)$. Other texture analysis parameters both in arterial and venous phase showed no significant difference for discriminating these two group renal tumors.

\section{Chrce Vs Ro}

The entropy of RO was significantly higher than that of chRCC both in arterial and in venous phase, with the AUC of 0.85 and 0.84 , respectively. The kurtosis of RO (1.24 \pm 1.50$)$ was significantly lower than that of chRCC $(4.97 \pm 6.91)(p \otimes 0.05)$ in the arterial phase with the AUC of 0.84 (sensitivity $91 \%$, specificity $74 \%$ ), while there was no significant difference in the venous phase ( $p \otimes 0.05)$. Other texture analysis parameters showed limited value in identifying chRCC from RO (Fig. 3, Fig. 4 and Fig. 5).

\section{Discussion}


In this study, we found texture analysis parameters mean, 25th, 50th, 75th, inhomogeneity, and entropy of ccRCC were significantly higher than those of chRCC, while the kurtosis was significantly lower than that of chRCC, which was in line with the previous study[11]. However, several previous studies have just described the value of texture analysis in discriminating chRCC from other renal tumors, not for discriminating ccRCC and chRCC. Yu H found that among the histogram parameters, only STD 5 parameter showed the discriminator with an AUC of 0.76 between chRCC and other renal tumors, while the others showed poor to fair discriminators of them[8]. Histogram analysis explained only about $64 \%$ of texture difference between chRCC from other renal tumors[11]. Although chRCC has substantial overlaps in enhancement with ccRCC, chRCC tends to show lower enhancement than ccRCC and more homogenous[9]. Entropy reflects the complexity of the image, kurtosis represents the peakedness or sharpness of the pixel distribution and the inhomogeneity shows the unevenness of the pixel distribution[7].ChRCC is most likely to be a homogeneous mass with a relatively hypovascular enhancement compared to ccRCC, and often a peak attenuation shows in the arterial or venous, on the contrary, ccRCC often demonstrates a peak enhancement in the arterial phase and with washout in the venous phase. However, chRCC has a substantial overlap in appearance compared with ccRCC $[12,13]$. So, our result could provide great help for discriminating homogeneous ccRCC from chRCC.

Central scar and segmental enhancement inversion were considered to be an unreliable and controversial feature to differentiate RO from $\operatorname{ccRCC}(9,14)$. Sasaguri, $\mathrm{K}$ combined the imaging features including tumor CT attenuation values and texture parameters (heterogeneity and skewness) to differentiate RO from RCC on biphasic contrast-enhanced CT, and the result showed this method could differentiate RO from ccRCC and other subtypes of RCC with an AUC of 0.82. Varghese, B. A. found that histogram analysis only explained about $74 \%$ of the textural difference between $\mathrm{RO}$ and the other tumors and the full texture model had an AUC of 0.87 for distinguishing RO from ccRCC[11]. Also, the heterogeneity showed to be an important parameter in renal tumor identification, and RO tended to be more homogenous than RCC[15]. Yu, H. showed the GLCM contrast of machine-based learning was a very good discriminator with an AUC of 0.80 , kurtosis and skewness were excellent discriminators of cCRCC from RO with AUCs of 0.93 and 0.91 , while in this study the author drew the ROI including the entire tumor and cystic necrosis of ccRCC was not excluded[8]. Also, there were challenges in differentiating ccRCC from RO because of the similar ADC values in the renal MR images[16]. Differentiation of RO from homogenous ccRCC still has challenges, especially from the homogeneous ccRCC. In our study, only the inhomogeneity showed limited diagnostic value in the arterial phase, while other parameters both in the arterial and venous phase showed limited help for diagnosis, these results were consistent with many literature reports.

It was difficult to differentiate RO from chRCC because of the cell origin, even with core needle biopsy. Entropy in the arterial and venous phase, kurtosis in the arterial demonstrated good diagnostic performance for discriminating chRCC from RO in our study. This was inconsistent with the results of the previous study, and $\mathrm{Yu}, \mathrm{H}$. showed that only histogram parameter median performed a very good discriminator of chRCC from RO with an AUC of 0.88 , while other parameters were poor to fair discriminators of them[8]. Varghese B A observed that the full texture model showed excellent discrimination power in differentiating RO from chRCC with an AUC of 0.94 [11]. But the study above was performed with complicated calculation and the entropy calculation based on ordinary CT images was simple and convenient, and could objectively quantify the heterogeneity of tumor[7]. Combining DWI and contrast-enhanced MRI images might assist in differentiating these two tumors with an AUC of 0.93 and a higher specificity[10].

There were several limitations in this study. Firstly, this was a single-center retrospective study, and results need to further prospective validation with large sample size. Secondly, the sample size of RO was relatively small, and limited subtypes of the renal tumor were included in this study. Thirdly, interobserver consistency analysis was not performed in this study, because a study had shown that the whole tumor texture analysis had better interobserver agreement than using a single slice ROI [16]and there was excellent interobserver agreement in several whole-tumor texture analysis studies[17].

\section{Conclusions}


The whole-tumor CT texture analysis as a quantitative biomarker could be a useful tool for discriminating the solid renal tumor on contrast-enhanced CT images in differentiating cCRCC from chRCC, chRCC from RO, especially in the arterial phase. Otherwise, the result showed that texture analysis still has challenges and provided limited help in distinguishing homogeneous ccRCC from RO.

\section{Abbreviations}

RCC: renal cell carcinoma; OR: renal oncocytoma; CTTA: CT texture analysis; ROI: region of interest; ROC: receiver operating characteristic curve; AUC: area under the receiver operating characteristic curve.

\section{Declarations}

\section{Ethics approval and consent to participate}

This retrospective study and all experimental protocols were approved the Ethics Committee of Tongji Hospital, Tongji Medical College, Huazhong University of Science and Technology (TJ-IRB20210514), and all methods were carried out in accordance with relevant guidelines and regulations. Due to the retrospective study design, written informed consent was waived.

\section{Consent for publication}

Not applicable.

\section{Availability of data and materials}

All data generated or analyzed during this study are included in this published article and all raw data were uploaded as a supplementary file.

\section{Competing interests}

The authors declare that they have no competing interests.

\section{Funding}

This work was supported by the National Natural Science Foundation of China (NO. 82071889).

\section{Authors' contributions}

Yonghua Niu conceived this study. Cui Feng searched the clinical data. Shichao Li analyzed the image data, Xiaoyan Meng analyzed the data and draft the manuscript. All authors interpret the data, revised the manuscript, and approved the final version.

\section{Acknowledgements}

Not applicable.

\section{References}

1. Motzer RJ, Jonasch E, Agarwal N, Bhayani S, Bro WP, Chang SS, Choueiri TK, Costello BA, Derweesh IH, Fishman M, et al. Kidney Cancer, Version 2.2017, NCCN Clinical Practice Guidelines in Oncology. J Natl Compr Canc Netw. 2017; 15(6):804-834. 
2. Tang Z, Yu D, Ni T, Zhao T, Jin Y, Dong E. Quantitative Analysis of Multiphase Contrast-Enhanced CT Images: A Pilot Study of Preoperative Prediction of Fat-Poor Angiomyolipoma and Renal Cell Carcinoma. AJR. American journal of roentgenology. 2020; 214(2):370.

3. Scrima AT, Lubner MG, Abel EJ, Havighurst TC, Shapiro DD, Huang W, Pickhardt PJ. Texture analysis of small renal cell carcinomas at MDCT for predicting relevant histologic and protein biomarkers. Abdom Radiol (NY). 2019; 44(6):19992008.

4. Bektas CT, Kocak B, Yardimci AH, Turkcanoglu MH, Yucetas U, Koca SB, Erdim C, Kilickesmez O. Clear Cell Renal Cell Carcinoma: Machine Learning-Based Quantitative Computed Tomography Texture Analysis for Prediction of Fuhrman Nuclear Grade. Eur Radiol. 2019; 29(3):1153-1163.

5. Suarez-lbarrola R, Hein S, Reis G, Gratzke C, Miernik A. Current and future applications of machine and deep learning in urology: a review of the literature on urolithiasis, renal cell carcinoma, and bladder and prostate cancer. World J Urol. 2019.

6. Deng Y, Soule E, Samuel A, Shah S, Cui E, Asare-Sawiri M, Sundaram C, Lall C, Sandrasegaran K. CT texture analysis in the differentiation of major renal cell carcinoma subtypes and correlation with Fuhrman grade. Eur Radiol. 2019; 29(12):6922-6929.

7. Feng Z, Shen Q, Li Y, Hu Z. CT texture analysis: a potential tool for predicting the Fuhrman grade of clear-cell renal carcinoma. Cancer Imaging. 2019; 19(1):6.

8. Yu H, Scalera J, Khalid M, Touret A, Bloch N, Li B, Qureshi MM, Soto JA, Anderson SW. Texture analysis as a radiomic marker for differentiating renal tumors. Abdom Radiol. 2017; 42(10):2470-2478.

9. Sasaguri K, Takahashi N. CT and MR imaging for solid renal mass characterization. Eur J Radiol. 2018; 99:40-54.

10. Zhong Y, Wang H, Shen Y, Guo A, Wang J, Kang S, Ma L, Pan J, Ye H. Diffusion-weighted imaging versus contrastenhanced MR imaging for the differentiation of renal oncocytomas and chromophobe renal cell carcinomas. Eur Radiol. 2017; 27(12):4913-4922.

11. Varghese BA, Chen F, Hwang DH, Cen SY, Desai B, Gill IS, Duddalwar VA. Differentiation of Predominantly Solid Enhancing Lipid-Poor Renal Cell Masses by Use of Contrast-Enhanced CT: Evaluating the Role of Texture in Tumor Subtyping. AJR Am J Roentgenol. 2018; 211(6):W288-W296.

12. Yang G, Gong A, Nie P, Yan L, Miao W, Zhao Y, Wu J, Cui J, Jia Y, Wang Z. Contrast-Enhanced CT Texture Analysis for Distinguishing Fat-Poor Renal Angiomyolipoma From Chromophobe Renal Cell Carcinoma. Mol Imaging. 2019; 18:1329152615.

13. Raman SP, Johnson PT, Allaf ME, Netto G, Fishman EK. Chromophobe renal cell carcinoma: multiphase MDCT enhancement patterns and morphologic features. AJR. American journal of roentgenology. 2013; 201(6):1268.

14. Schieda N, Mcinnes MDF, Cao L. Diagnostic accuracy of segmental enhancement inversion for diagnosis of renal oncocytoma at biphasic contrast enhanced CT: systematic review. Eur Radiol. 2014; 24(6):1421-1429.

15. Sasaguri K, Takahashi N, Gomez-Cardona D, Leng S, Schmit GD, Carter RE, Leibovich BC, Kawashima A. Small ( $4 \mathrm{~cm})$ Renal Mass: Differentiation of Oncocytoma From Renal Cell Carcinoma on Biphasic Contrast-Enhanced CT. AJR. American journal of roentgenology. 2015; 205(5):999.

16. Hötker AM, Mazaheri Y, Wibmer A, Zheng J, Moskowitz CS, Tickoo SK, Russo P, Hricak H, Akin O. Use of DWI in the Differentiation of Renal Cortical Tumors. AJR. American journal of roentgenology. 2016; 206(1):100.

17. Lu J, Hu D, Tang H, Hu X, Shen Y, Li Z, Peng Y, Kamel I. Assessment of tumor heterogeneity: Differentiation of periampullary neoplasms based on CT whole-lesion histogram analysis. Eur J Radiol. 2019; 115:1-9.

\section{Figures}




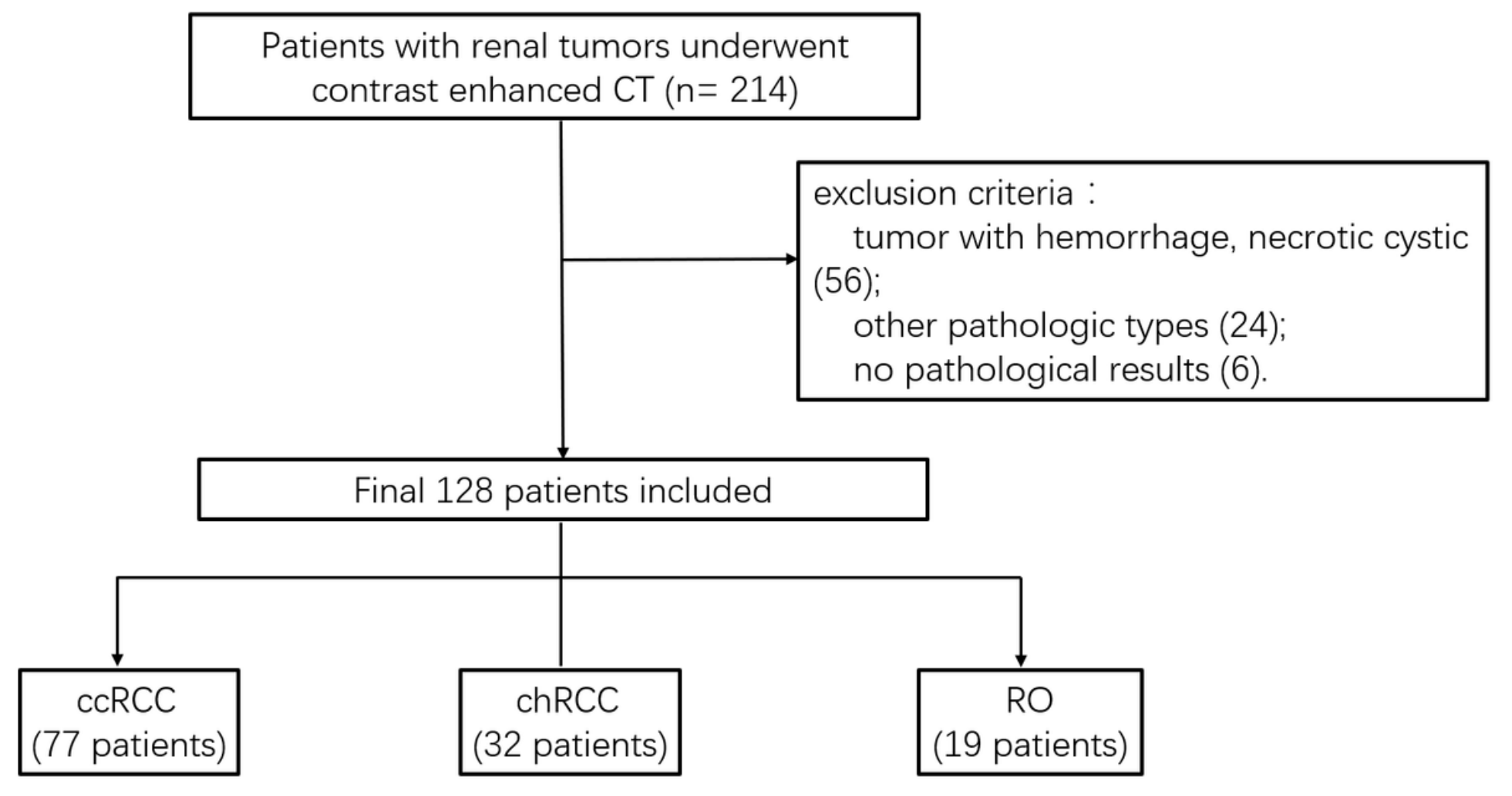

Figure 1

Flowchart of the study population.
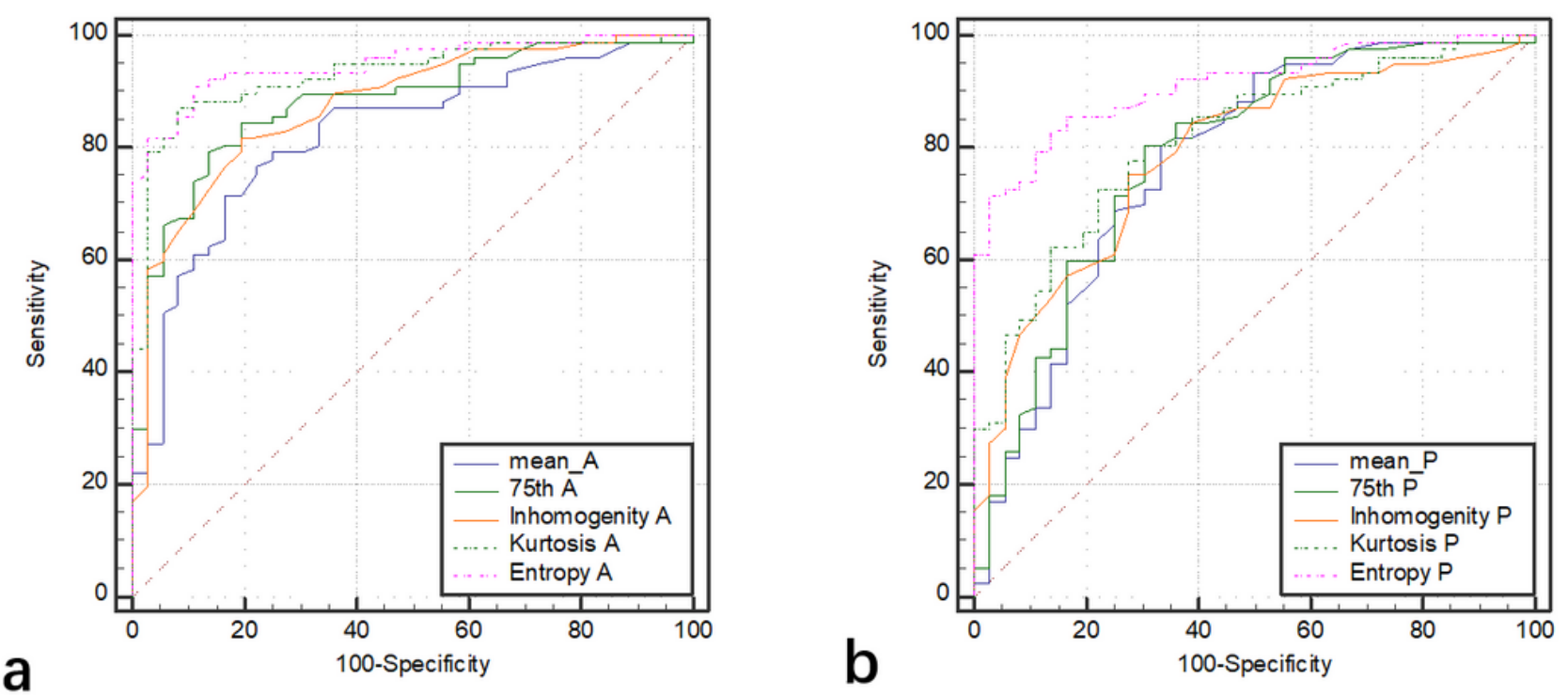

Figure 2

The ROCs of texture analysis parameters in differentiating ccRCC from chRCC in arterial and venous phase.

Figure 3 
A 53-year-old female patient found a tumor on the right kidney in a physical examination and pathologically confirmed as clear cell renal cell carcinoma (ccRCC) and the pathology of liver tumor showed as hepatic vascular smooth muscle lipoma. The pictures in the first and second row showed the arterial and venous phase scan respectively, and the texture analysis parameters were as followed: $119 \mathrm{HU}, 0.030,-0.39,-0.05$ and 4.18 in the arterial, $105 \mathrm{HU}, 0.020,-0.61,1.22$ and 3.74 in the venous phase (mean, inhomogeneity, skewness, kurtosis and entropy).

\section{Figure 4}

A 48-year-old female patient found a tumor on the left kidney and pathologically confirmed as chromophobe renal cell carcinoma (chRCC). The pictures in the first and second row showed the arterial and venous phase scan respectively, and the texture analysis parameters were as followed:81HU, $0.014,0.29,-1.35$ and 3.49 in the arterial, $77 \mathrm{HU}, 0.015,0.10,0.62$ and 3.56 in the venous phase (mean, inhomogeneity, skewness, kurtosis and entropy).

\section{Figure 5}

A 40-year-old female patient found a tumor on the right kidney and pathologically confirmed as renal oncocytoma (RO). The pictures in the first and second row showed the arterial and venous phase scan respectively, and the texture analysis parameters were as followed:272HU, 0.042, $-0.73,0.89$ and 3.94 in the arterial, $154 \mathrm{HU}, 0.018,-0.45,2.91$ and 3.53 in the venous phase (mean, inhomogeneity, skewness, kurtosis and entropy). 